\title{
Age Differences in the Identification of Concepts of the Natural Language ${ }^{1}$
}

\author{
Carole J. Quarterman and Klatis F. Riegel \\ University of Michigan
}

\begin{abstract}
Twenty-four school children each at the age levels of about $6,9,12$, and 15 years were tested on four types of experimentally determined conceptual clues. The task was to retrieve 16 stimuli that were originally used in a restricted-association study. The number of correct answers was found to increase as a function of age, clue type, and the number of clues given. The results indicate that while different explanatory techniques for words are not necessary for different age levels, the type of relationship and the number of clues given are important determinants for concept identification.
\end{abstract}

Traditionally, words are defined either generically by a superordinate and an attribute, e.g., a zebra is an animal with stripes, or typologically by similars or coordinates, e.g., a zebra is like a horse. In the past, differcnecs in efficiency of these definitions have not becn taken into account and their dependency on the words to be defined or the persons to be informed have not been studied. Moreover, no relations and no combinations other than superordinates/attributes have been explored, although at least the similarity relation has been available for consideration. The present paper focuses on age differences in the identification of concepts or of the meaning of words by applying different types and different combinations of word relations.

In a study of restricted word associations, Riegel, Riegel, Quarterman, and Smith (1968) found that word relations are treated more independently by older children and, when explaining words, young children will enumerate parts or qualities of the denoted objects but, with increasing age, will use similars or superordinates and will combine these terms rather than apply them in isolation. These findings imply that if the word association process is reversed (i.e., if an associative response is given as a clue word and $S_{s}$ are asked to identify the original stimulus as the target word) performance should vary at least according to five

${ }^{1}$ This study has been supported by Grant 1P01HD01368-04, United States Public Health Service. The authors are indebted to Mrs. Marilyn T. Zivian and Dr. Ruth M. Riegel for their assistance. 
factors: (1) age of $S \mathrm{~s},(2)$ target words (original stimuli), (3) associative frequency with which target words (original stimuli) elicit clue words (original responses), (4) number of clue words given, and (5) type of relations combined.

Zivian and Riegel (1968) used this technique with college students and observed that Ss responded correctly more often (1) when similar rather than location clues were given; (2) when high rather than low associates were used as clues; (3) the more clues they were given.

In the present study we investigated how four different age groups (first, third, sixth, and ninth graders) would perform on this task when four types of relations are either presented singularly or in pairs. Holding association strength between target and clue word constant, the main predictions are: (1) All age groups will produce about the same number of correct answers when given part and location clues but, increasing with age, they will produce more correct answers with superordinate and similar clues. Subsequently, younger children are expected to be relatively better with part and location clues and older children with superordinate and similar clues. (2) Because of the greater variability in the type of information provided, mixed clues will be more efficient than either the combination of parts and locations or of superordinates and similars. Because of the increase with age in efficiency of both similars and superordinates, also their combination is expected to increase in efficiency. (3) Regardless of age, $S \mathrm{~s}$ are expected to perform better with double than with single clues.

\section{PROCEDURES}

Material. Words were chosen from the Michigan restricted association norms (Riegel, 1965) in which 100 college students responded to 200 stimuli each under 16 different instructions. The 16 tasks have been divided into three main categories: logical, grammatical, and infralogical. In the present study, the concern is only with the infralogical tasks of parts and locations and the logical tasks of superordinates and similars. The term infralogical (Flavell, 1963) refers to a physical relationship in time or space. For example, the part тоE has a spatial relationship to the whole denoted by the original stimulus FоOт. A logical relationship on the other hand implies verbal abstractions. For example, a superordinate denotes an abstract category, such as ANIMAL, under which a number of items can be classified on the basis of common qualities, functions or parts, for instance zEBRA, IIORSE, cow.

Target words. On the assumption that the responses of college students represent a standard which children will increasingly approximate, 16 noun stimuli were selecled as target words from the norms. The stimuli 
had been used successfully in an earlier study of restricted associations by Riegel et al. (1968) using third and sixth grade students and occurred in a child's vocabulary by 6 years of age (Rinsland, 1945). Zivian and Riegel (1968) also used the same 16 stimuli.

Clue words. Superordinates, similars, parts, or locations that did not occur as primary or high-frequency responses to the same stimulus under any of the other three tasks of restricted associations were selected as clue words for each of the 16 target words. The mean associative frequency varied slightly between the four tasks and was lower for parts $(38 \%)$, locations $(36 \%)$, and similars $(34 \%)$, than for superordinates $(48 \%)$.

Clue combinations. The four types of clues were combined into all possible pairs. No pair included the same clue twice. The pairs consisting of a superordinate and a similar clue and the pairs consisting of a part and a location clue will be called 2-way logical or 2-way infralogical clues, respectively. Any single clue will be called either a 1-way logical (superordinate or similar) or a 1-way infralogical (part or location) clue. The combinations of logical and infralogical clues will be called mixed clues.

Presentation. The clue words were inserted into the following sentence frames:

I am looking for something which

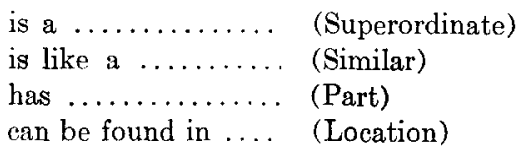

The sentences for the clue combinations were derived by joining the dependent clauses of the single-clue frames, as for instance:

I am looking for something which is a $\ldots \ldots \ldots \ldots$ and has $\ldots \ldots \ldots \ldots$

The experiment was presented to the children as a word-guessing game. Each sentence was read to $S$ and $E$ recorded the answers on the test forms. If requested, the sentences were repeated once or twicc, so that there was no time pressure. $E$ recorded the time used by $S$ for the completion of the whole list of items. Ss were not told whether their answers were correct or not.

Ss were required to identify the same target words ten times, however, each time under a different clue combination. The 160 combinations were randomized for each $S$ within the five categories: logical 1-way (32), logical 2-way (16), infralogical 1-way (32), infralogical 2-way (16), and mixed (64). The order of presentation of these five categories was counterbalanced to yield $\mathbf{2 4}$ different test forms. Each $S$ within an age group 
received a different test form. Ss were not informed that the same tirget words were to be identified repeatedly. Since the list of 16 target words seems long enough when embedded ten times into the total list of 160 different clue combinations, the method of repeated measurements was preferred over one using different samples of Ss. Moreover, our method allows for a more comprehensive analysis of individual differences and serial order effects.

Subjects. Ninety-six school children were tested in a mobile trailer that was taken to the school sites in Ypsilanti, Michigan. According to the reports from the school psychologist, the children were recruited from classes of about average intellectual and socioeconomic standards. There were 12 boys and 12 girls in each of the following age and grade levels: 6.4-year olds, first grade; 8.8-year olds, third grade; 11.8-year olds, sixth grade; 14.8-year olds, ninth grade.

\section{RESULTS AND DISCUSSION}

The occurrences of the 16 target words in either singular or plural iorm were counted as correct answers. No synonyms or phrases including the target words were regarded as correct. The percentages of correct. answers given in Table 1 increase steadily up to the sixth grade. The percentages of the ninth graders are lower though they do not drop to the levels of the first and third graders. The results were evaluated in the following three variance analyses corresponding to the three hypotheses stated in the introduction.

In the first analysis, comparisons were made between the four types of single clues and grades (see upper part of Table 1). Significant main effects were found for age groups $(F=15.1 ; d f=3 / 92 ; p<.01)$ and clue types $(F=313.4 ; d f=3 / 276 ; p<.01)$. There was also a significant interaction $(F=4.4 ; d f=9 / 276 ; p<.01)$ indicating, in particular, deviations of the ninth graders from the other $S$ s.

For all grades similars proved to be the most and superordinates the least useful single clues. Parts and locations were of medium efficiency. These results differ from those obtained in a study of college students (Zivian and Riegel, 1968), in which the order of correct responses was found to be similars, superordinates, parts, and locations, and in which, thus, Ss performed consistently better with single logical than with single infralogical clues. In order to determine whether the last finding could be generalized to the four grade levels of the present study, $t$-test comparisons between the pooled logical and the pooled infralogical clues were made. Although $S$ s at all grade levels achieved higher average scores on the former, none of the differences was significant. Furthermore, the combined sixth and ninth graders performed significantly 
TABLE 1

Percentage of Correct R.feponses as a Ftunction of Types of Crujes, Number of Cuues, and Grade Levels; Percentage of Errors Listed in the Norms, Percentage of Omission and Test Duration as a Function of Grade Levels

\begin{tabular}{lrrrr}
\hline & \multicolumn{4}{c}{ Grade level } \\
\cline { 2 - 5 } & 1st & \multicolumn{1}{c}{$3 \mathrm{rd}$} & 6 th & 9 th \\
\hline Single clues average & 20.0 & 26.0 & 36.2 & 33.6 \\
Similars & 41.4 & 50.8 & 73.2 & 65.9 \\
Parts & 21.6 & 25.0 & 33.6 & 32.3 \\
Locations & 9.4 & 14.8 & 19.3 & 19.3 \\
Superordinates & 7.6 & 13.3 & 18.8 & 16.8 \\
Double clues average & 26.9 & 41.0 & 59.3 & 55.2 \\
Similars-locations & 35.4 & 54.1 & 80.2 & 70.5 \\
Similars-parts & 34.6 & 52.6 & 73.6 & 69.7 \\
Similars-superordinates & 34.1 & 50.3 & 69.8 & 67.7 \\
Parts-locations & 24.2 & 33.9 & 53.4 & 52.9 \\
Parts-superordinates & 19.5 & 30.2 & 41.4 & 41.6 \\
Locations-superordinates & 13.8 & 24.7 & 37.2 & 28.6 \\
\% errors & 23.3 & 31.9 & 41.9 & 52.4 \\
\%omissions & 2.4 & 1.1 & .9 & .4 \\
Test duration (minutes) & 32.6 & 33.0 & 28.7 & $\mathbf{1 8 . 3}$ \\
\hline
\end{tabular}

better than the combined first and third graders on both the logical and infralogical clues $(t=13.3$ and $t=7.3$, respectively; $d f=126 ; p<$ .001 for both groups). For these reasons, we abandoned the hypothesis that youngcr children would perform relatively better with infralogical and older children with logical clues, and that there would be little increase with age in the efficiency of the infralogical clues.

In the second analysis, comparisons were made between all six types of double clues and grades (see second part of Table 1). Again both main effects for age and clue types $(F=9.0 ; d f=3 / 92 ; p<.01$ and $F=576.7 ; d f=5 / 480 ; p<.01$, respectively) and interaction $(F=$ $152.0 ; d f=5 / 480 ; p<.01)$ were found to be significant. The latter again seems to indicate particular deviations of the ninth graders from the other Ss.

The order in efficiency of the clue combinations is the same for all grade levels, whereby the 2-way logical and the 2-way infralogical clues take the middle positions among the six combinations. Mixed clues are consistently superior when they include similars, but inferior when they include superordinates. When $t$-test comparisons were made between the average scores of the double clues, no significant differences 
were found for any grade level between the mixed and 2-way infralogical clues nor between the 2-way logical and 2-way infralogical clues. For all grades 2-way logical clues yielded significantly higher scores than mixed clues $\left(t_{1}=2.08, t_{3}=2.18, t_{6}=2.04, t_{9}=3.91 ; d f=78\right.$ for all grades; $p<.05$ for all grades except $\left.p_{9}<.01\right)$. On the basis of these comparisons, we rejected the hypothesis that mixed clues are
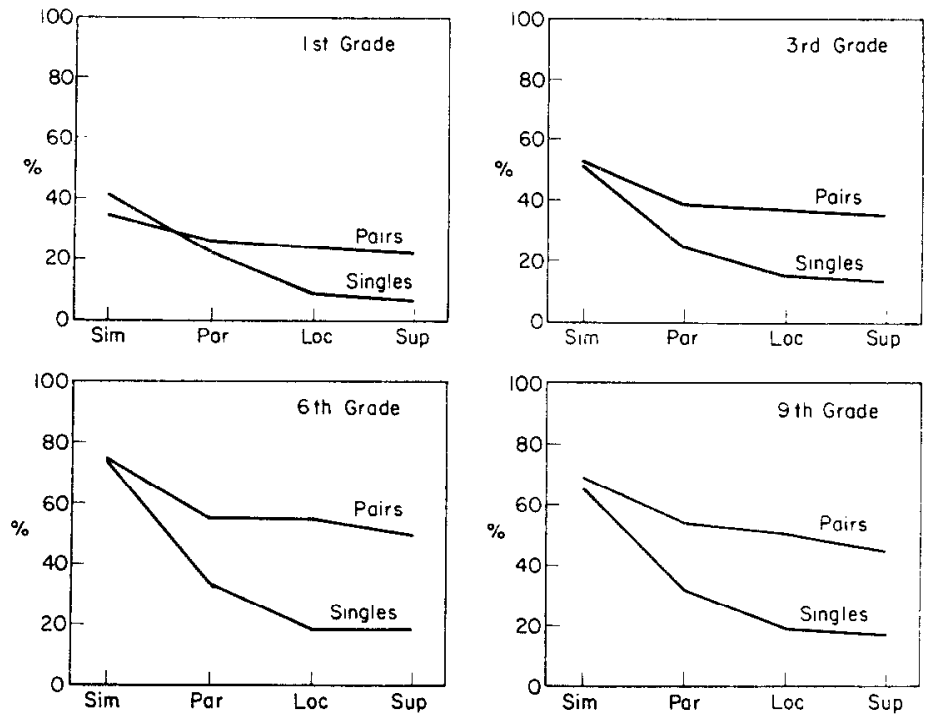

FIa. 1. Percentage of correct identification of stimuli when clues were given either singly or in pairs with any of the other three clues.

The different clue types (logical, infralogical, and mixed) were counterbalanced so that each could appear in one of three positions for the first time. When the percentages of correct responses were averaged for each position ignoring clue types, the first graders performed at a constant level. All the other grades showed improvements for each successive position. IIowever, only the difference between pusition 1 and 3 for the ninth graders was found to be significant $(t=2.3, d f=46 ; p<.05$ ). However, this learning effect was not great enough to enable the ninth graders in their last trials to perform better with 1-way than with 2-way clues. Two-way clues always yielded more correct answers than 1-way clues, regardless of their positions.

Since only those answers that were identical with the stimuli of the norms were considered to be correct, any error might represent a rather reasonable substitute for its target word. In an investigation of this problem, we determined how often any particular error was listed as a response and, thus, could be considered as related in a rational manner to its target word (original stimulus) under any of the 16 tasks of the Michigan restricted-association norms. As shown in Table 1 , the percentages of the errors that could be located in the norms increase steadily with grades. Finally, the percentages of omissions are negligible and-like the time needed to complete the tasks-decreases with advancing age. 
more useful than pairs of either logical or infralogical clues. Contrary to our expectations, not only 2-way logical but all other double clues increase in efficiency with age.

In the third analysis, comparisons were made between the number of clucs and grades (sce first and sixth lines of Table 1). Significant effects were found for grades $(F=30.8 ; d f=3 / 92 ; p<.01)$, number of clues $(F=440.0 ; d f=1 / 92 ; p<.01)$ and their interaction $(F=14.7 ; d f=$ $3 / 92 ; p<.01)$. The interaction is due to the markedly smaller differences between double and single clues for the first graders as shown in Fig. 1 and as also indicated by the differences in $t$ values $\left(t_{1}=3.7, t_{3}=\right.$ $8.1, t_{6}=7.2, t_{9}=8.1 ; d f=158 ;$ and $p<.001$, for all grades). In particular, the significant interaction is related to the differential efficiency of single similars. As can be seen in Table 1, for first graders, single similars are more efficient than any double clues. For the older $\mathrm{Ss}_{\mathrm{s}}$, single similars surpass only the last three or four double clues listed in the table. In some instances, also single parts are more efficient than double clues, however, an age effect is less clearly delineated in this case.

A different way of analyzing the interaction effect is presented in Fig. 1. IIere, the percentages of correct responses to each of the single clues are compared with the average percentages for the same clues when they were presented with the other three in double clue presentations. Second clues, when added to single parts, locations, or superordinates, always improve the performance markedly. With the exception of the first graders, similars when used in combination with other elues always yield the highest percentages. However, these percentages surpass only slightly those of single similars and, in the case of the first graders, do not even reach this level. In general, the results nevertheless support the hypothesis that double clues are more efficient than single clues.

\section{CONCLUSIONS}

The present experiment imitates situations in which linguistic information is passed on or exchanged between individuals. In particular, if in such a situation, the meaning of a word is to be explained to a child, the teacher may rely on other words (clues) semantically related to the word to be explained (target). In order to explain what a zEBRA is, the informer may point out, for instance, that it is an ANIMAL (superordinate), has STRIPES (part), or is like a HORSE (similar). The informed will then substitute the name zEBRA for the intersect of the three semantic relations activated by the informer. The success with which the informed will acquire a sufficiently distinct notion of a zEBRA will depend in part on the informer's semantic strategy and, in part on 
the size and complexity of the individual's own verbal repertoire. The present experiment was concerned only with the former. It was assumed that the individual's verbal repertoire already included the target words. the clue words, and sufficiently clear conceptions of the semantic relations brought into focus by the informer.

The above interpretations imply semantic descriptions similar to those advanced in psychology and stated either in terms of association theory (Deese, 1962, 1965) or emphasizing response sets and categorizations (Riegel and Riegel, 1963). Also linguists are returning to the discussion of these problems (Katz and Fodor, 1963), but remain more interested in an analysis of linguistic competence than of the performing $S$ s and individual differences. Relying on "linguistic intuitions" and lexicographical descriptions, Katz and Fodor have emphasized the semantic relations of superordination and similarity and, thus, notions implied in classical generic and typological definitions. By and large, other relations and especially those infralogical relations characteristic of the denoted objects rather than the denoting words, have been disregarded in their interpretations.

According to our results, generic definitions are not the most efficient procedures for the identification of words or concepts. Definitions by similars lead more readily to correct responses. Even though Ss performance was better when double instead of single elues were given, the addition of other clues to similars did not necessarily produce marked improvements. Similars were not only the most useful single clues, but the performance of first graders was best for single similar clues than for any other single or double clues. This exceptional finding might be explained by a lack in short-term retention and the inability of first graders to process simultaneously the two types of information provided by paired clues. Comparable interpretations are implied in McLaughlin's (1963) attempt to explain differences in cognition of children at various developmental stages by differences in their ability to perform simultaneously different categorizations and, ultimately, by the growth of their retention span.

Although there was a considerable increase in performance, the order of both single and double clues in terms of their usefulness for the identification of the target words was precisely the same for all grade levels. Thus, there seems to be no need to use different principles for explaining concepts at various grade levels. Explanations at the younger age levels should be limited, however, to a few discret hints rather than to simultaneous presentations of different clues which might be too heavy a burden on the child's storage and combinatory abilities. 


\section{REFERENCES}

DeEse, J. On the structure of associative meaning. Psychological Review, 1962, 69, 161-175.

DeEse, J. The structure of associations in language and thought. Baltimore, Maryland: Juhus Hopkins Press, 1965.

Flaverl, J. H. The developmental psychology of Jean Piaget. Princeton, New Jersey: Van Nostrand, 1963.

KATz, .J., AND FoDOR, J. The structure of semantic theory. Language, 1963, 39, 170210.

McLaughuin, G. H. Psychologic: A possible alternative to Piaget's formulation. British Joumal of Educational Psychology, 1963, 33, 61-67.

RIEGEL, K. F. The Michigan restricted association norms. Report No. 3, USPHS Grant No. MH 07619, Department of Psychology, University of Michigan, 1965.

Riegiel, K. F., and Riegel, Ruth M. An investigation into denotative aspects of word meaning. Language and Speech, 1963, 6, 5-21.

Rieget, K. F., Riegel, Ruth M., Quarterman, Carole J., and Smith, Helen E. An analysis of difference in word meaning and semantic structure between four educational levels. Human Development, 1968, 11, 92-106.

Rinsland, H. D. A basic vocabulary of elementary school children. New York: Macmillan, 1945.

Zivian, Marilyn T., and Riegel, K. F. Word identification as a function of semantic clues and association strength. Journal of Experimental Psychology, 1968. 75, (in press). 